\title{
Study of Complications in Patients Using Removable Dentures Over Different Periods
}

\author{
Sviatoslav Rozhko*, Ivan Paliichuk
}

\begin{abstract}
The topicality of the problem is related to the ever-increasing volumes of provision of prosthodontic assistance to the patients with removable dentures. During the dental examination we've noted 300 patients, who used removable dentures, the increased number of complications because of these constructions' use. In particular, there is a growing number of patients who aren't using the already made removable dentures. A significant percentage of patients don't know or don't fully care properly for the existing constructions and the oral cavity itself. This leads to a number of serious complications. We consider perspective the identification of opportunities to reduce the number of complications and extend the period of the use of removable denture structures by improvement the techniques for selecting denture base materials depending on the microbiological status of the oral cavity.
\end{abstract}

Keywords

removable dental prostheses; complications; denture base materials

Ivano-Frankivsk National Medical University, Ivano-Frankivsk, Ukraine

*Corresponding author: rozhkosviatoslav@gmail.com

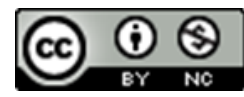

Copyright (C)Sviatoslav Rozhko, Ivan Paliichuk, 2019

\section{Problem statement and analysis of the latest research}

Providing prosthodontic assistance to the population using the removable dentures demonstrates constant dynamics to growth. Thus, according to researchers, the number of elderly patients who need to be made removable dental prostheses is $60 \%$ per 1000 of studied persons. Along with this, the number of patients who were diagnosed with various complications, is increasing (in $75 \%$ of the studied patients). Several researchers note the negative impact of the denture base materials on the microbiological status of the oral cavity. Research performed by a number of authors, showed significant deterioration in the hygienic condition of the oral cavity, that eventually leads to a reduction in terms of removable dentures' use. Unreasonable use and the use without proper control of the adhesive agents also lead to a number of serious complications.

The objective of the research is to study the nature of complications in patients during the use of removable dentures over different periods.

\section{Materials and Methods}

In order to study the complications of the use of removable dental prostheses, we've performed the dental examination of 300 patients, who applied to prosthetic dentistry clinic of IFNMU.

In terms of use, the number of patients was as follows: up to 6 months of the use of removable dentures - 103 patients, up to 2 years -101 patients, and after 3 years - 96 patients, respectively. The subject of study became the reason why patients appealed to the clinic. All patients were divided according to the age, timing of the use of removable dentures. There were studied the most 


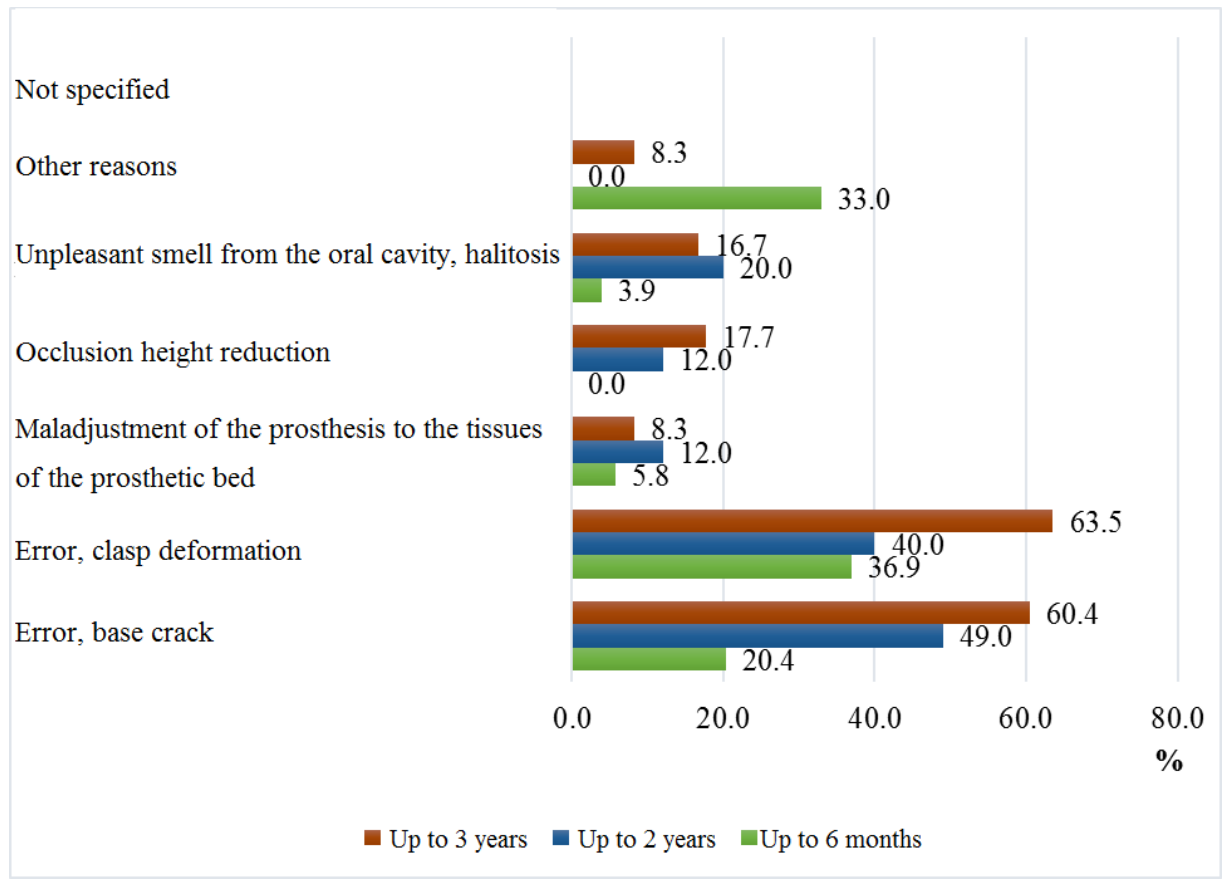

Figure 1. Reasons for patients to visit the clinic of orthopedic dentistry.

common causes why patients didn't use removable dental prostheses. Particular attention was paid to the hygienic condition of the oral cavity and to the removable dentures, impact on the patients' condition, their relation and knowledge of the necessity for hygienic care of the oral cavity and removable dentures. An important issue was the clarification of patients' stay on dispensary record. The results of examination were recorded in a specially made cards.

\section{Results and Discussion}

As a result of dental examinations, it was found that the most common cause of dental care encounter to prosthodontists was breaking and deformation of clasps. This complication was observed in $63.5 \%$ of studied patients after three-year period of use. High percentage of appeals was fixed due to breakage and cracks of bases of removable dentures. This complication has been observed in $60.4 \%$ of studied patients. In $20.0 \%$ - there was a bad breath after 2 years of the use of removable dental prostheses.

Reduction in bite height was recorded in $17.7 \%$ of studied patients, $12.0 \%$ of patients have complaints and discrepancy of denture to prosthetic bed tissues, which had a poor fixation of constructions' details. A significant number of patients, which is $33 \%$, had other reasons and used dentures for less than 6 months.

Age distribution was the following (Fig. 2).

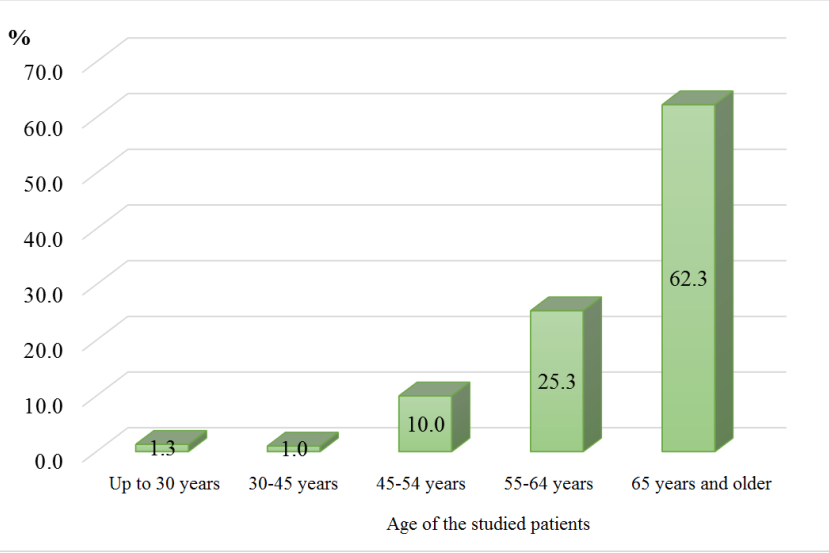

Figure 2. Distribution of patients according to the age.

There is a clear tendency to increase the number of patients who applied to the clinic of prosthetic dentistry because of the age (from $1.3 \%$ in the age group up to 30 years to $62.3 \%$ in the age group of 65 years and older). 
We've found a significant predominance of prosth- of their manufacture and the materials from which odontic re-treatment over primary during dental examination (Fig. 3).

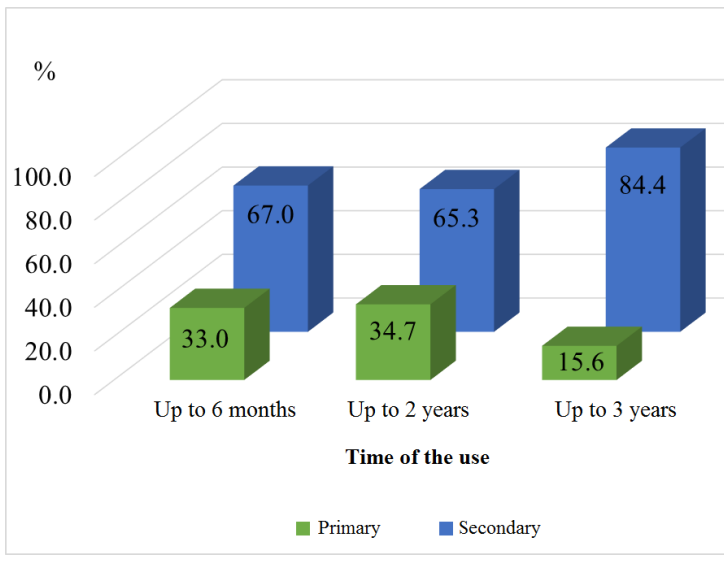

Figure 3. The order of orthopedic treatment.

Thus, in the period of use up to 6 months the ratio was from $33 \%$ to $67 \%$, in the period up to 2 years - from $34.7 \%$ to $65.3 \%$ and during the period of more than 3 years - from $15.6 \%$ to $84.4 \%$ of the studied patients, which indicates progressive increase of prosthodontic re-treatment.

The number of removable dentures showed the following dynamics (Fig. 4).

The partial removable laminar dentures were produced in considerably larger volumes and ranged from $53.3 \%$ to $71.9 \%$, whereas full dental prostheses from $11.5 \%$ to $28.9 \%$ of studied patients. This definitely indicates a permanent loss of supporting teeth and progression of dental diseases in the oral cavity.

The problem of extending the effective use of removable dentures is deeper and more complicated, because it combines not only the technologies they are made, but also the presence of prosthodontists, and perhaps the most important link is the patients themselves.

Fig. 5 shows the information on the provision of information and guidance by prosthodontists for patients.

The majority of prosthodontists provide advice and guidance to patients regarding the rules for removable dentures use. This percentage ranges from $87.4 \%$ to $97.7 \%$ of examined. From $2.3 \%$ to $12.6 \%$ of the studied patients were not informed, which converges with the number of patients who didn't use the manufactured dentures. These data are represented in the Fig. 6.

During the period of adaptation, which is the most important in prosthodontic treatment, $22.3 \%$ of patients don't use prostheses, in the period of up to 2 years, the percentage reduces twice and is $11.9 \%$, and in the period of over 3 years of use $4.2 \%$ of the examined. The obtained data confirm the depth of the problem, that must be solved in future.

The next stage of our observations was related to patients who followed the instructions of prosthodontists. These data are represented in the Fig. 7.

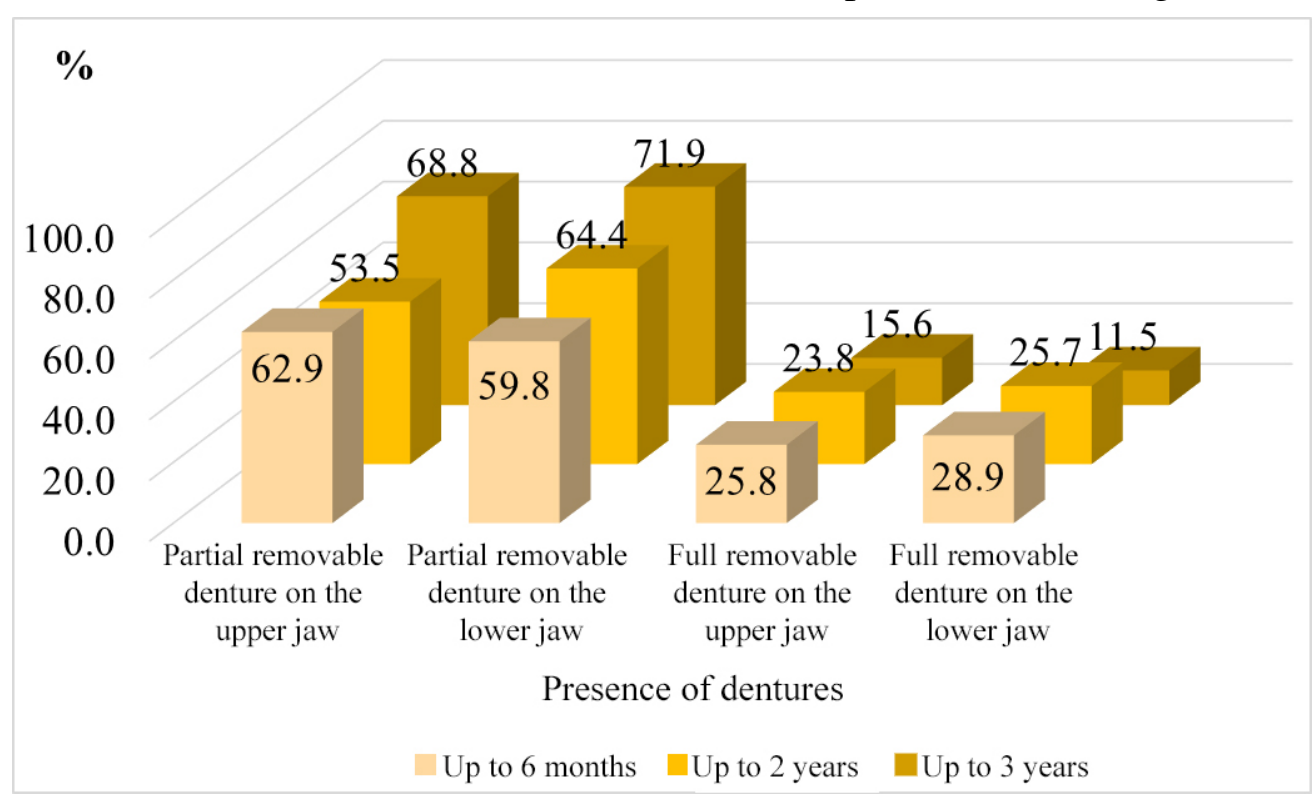

Figure 4. Laboratory indices of calcium metabolism in the examined patients. 


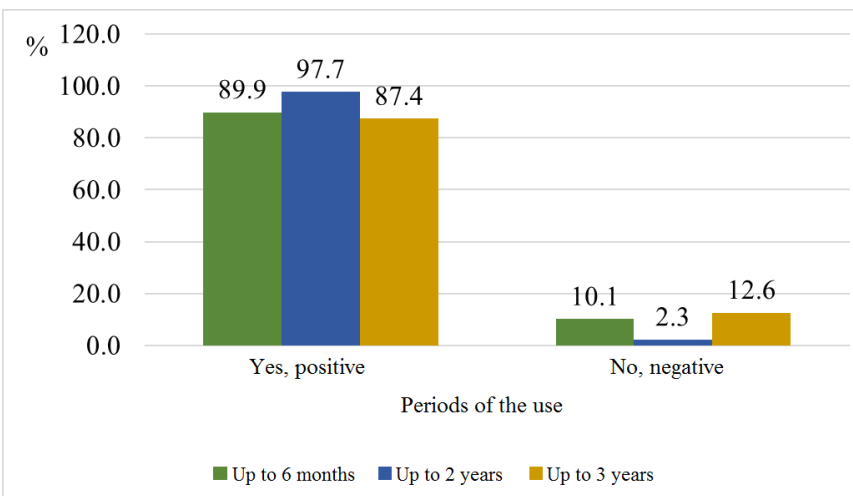

Figure 5. Provision of advice and guidance for patients by prosthodontists.

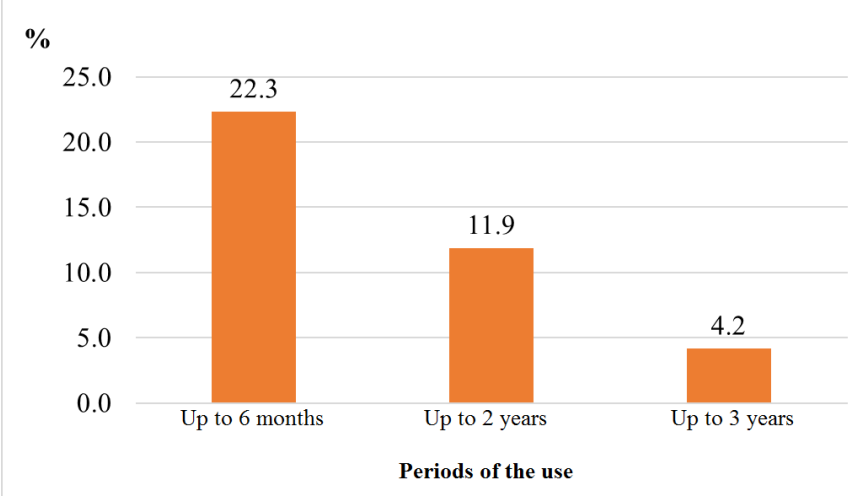

Figure 6. The number of patients who do not use manufactured orthopedic structures.

In the first place, that was found, it was an unpleasant smell from the oral cavity (halitosis was observed in $58.3 \%$ of studied patients who used dentures up to 3 years, in $88.6 \%$ in the period of up to 2 years). This index very reasonably characterizes the state of oral hygiene.

The state of oral hygiene cannot be separated from the hygienic state of the dental prostheses (Fig. 8).

Poor hygiene was indicated by a plaque on denture base, which was present from $28.3 \%$ to $73.3 \%$ of the studied. The next major point was the adjustment of the bases, which are depos for the accumulation of food residues and reproduction of microorganisms. This complication was observed in $20.0 \%$ to $68.3 \%$ of the studied. The minor processes of aging of denture base materials pointed out their significant change in color, which was observed in $17.7 \%$ of the studied patients. We've noticed normal condition of the denture base only in $1 \%$ of examined patients.

The quality and efficacy of the oral cavity care as a result of patients' survey showed that they give sufficient attention to this important component (Fig. 9), which does not coincide with the dental examination results and data.

From the received data from $60.7 \%$ to $96.2 \%$ of the interviewed patients clean the teeth and at the same time from $40.8 \%$ to $72.9 \%$ of patients do not indicate or do not care for the oral cavity.

In our opinion, the insufficient oral care, undifferentiated choice of denture base materials leads to imbalance of microbiological state of the oral cavity, which in turn leads to a reduction in the terms of effective use, and in many cases to the complete refusal to use removable denture structures.

\section{Conclusions}

With the increase in the number of removable dentures that are produced, the number of complications in patients is increasing too. There was a tendency to completely abandon the use of removable dentures. Possible causes of these complications have been identified.

\section{Prospects of Further Researches}

It is planned to further develop a differentiated choice of denture base materials depending on the microbiological condition of the oral cavity.

\section{References}

[1] Labunets VA, Diyeva TV, Diyev EV. Factors determining the population's necessity for a complete removable prosthesis. Odesky medychny visnyk. 2002; 6: 107-110.

[2] Paliychuk IV. Analysis of the use of different types of orthopedic structures and their effect on the oral cavity mucous membrane. Novyny stomatolohiyi. 2015; 2: 13-16.

[3] Mykhaylenko TM. Component analysis of the factors that determine and influence the hy- 


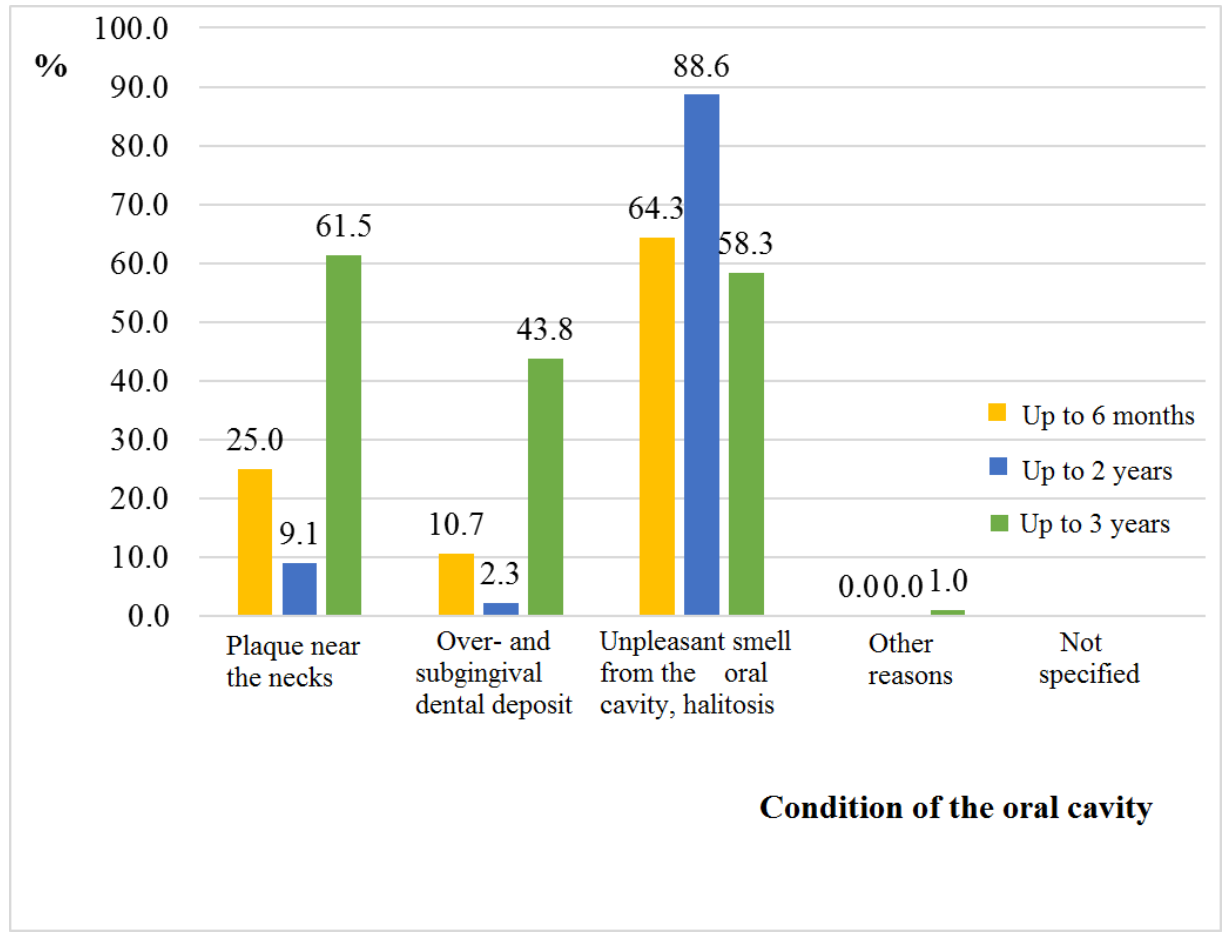

Figure 7. Hygienic condition of the oral cavity in patients using removable dentures.

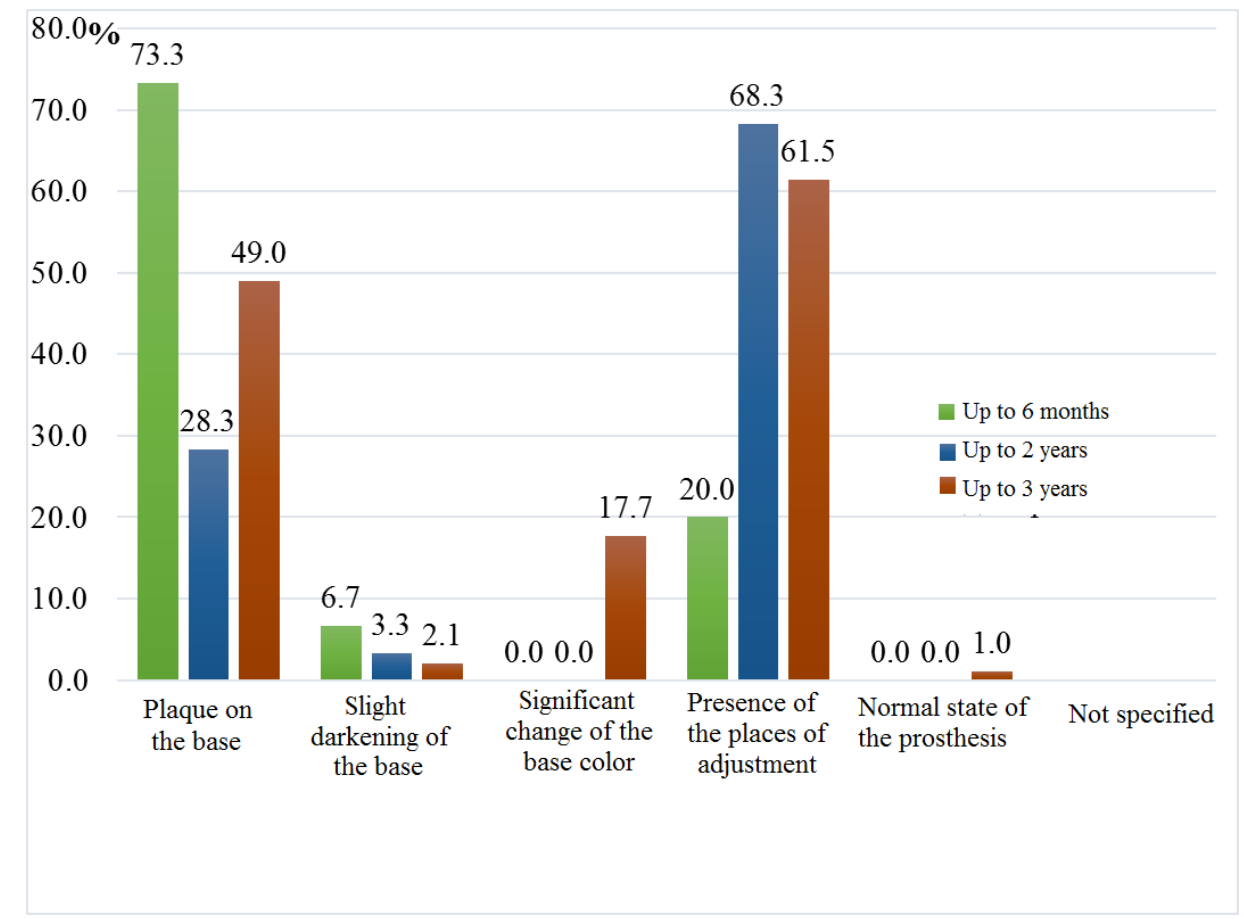

Figure 8. Hygienic condition of orthopedic structures. 


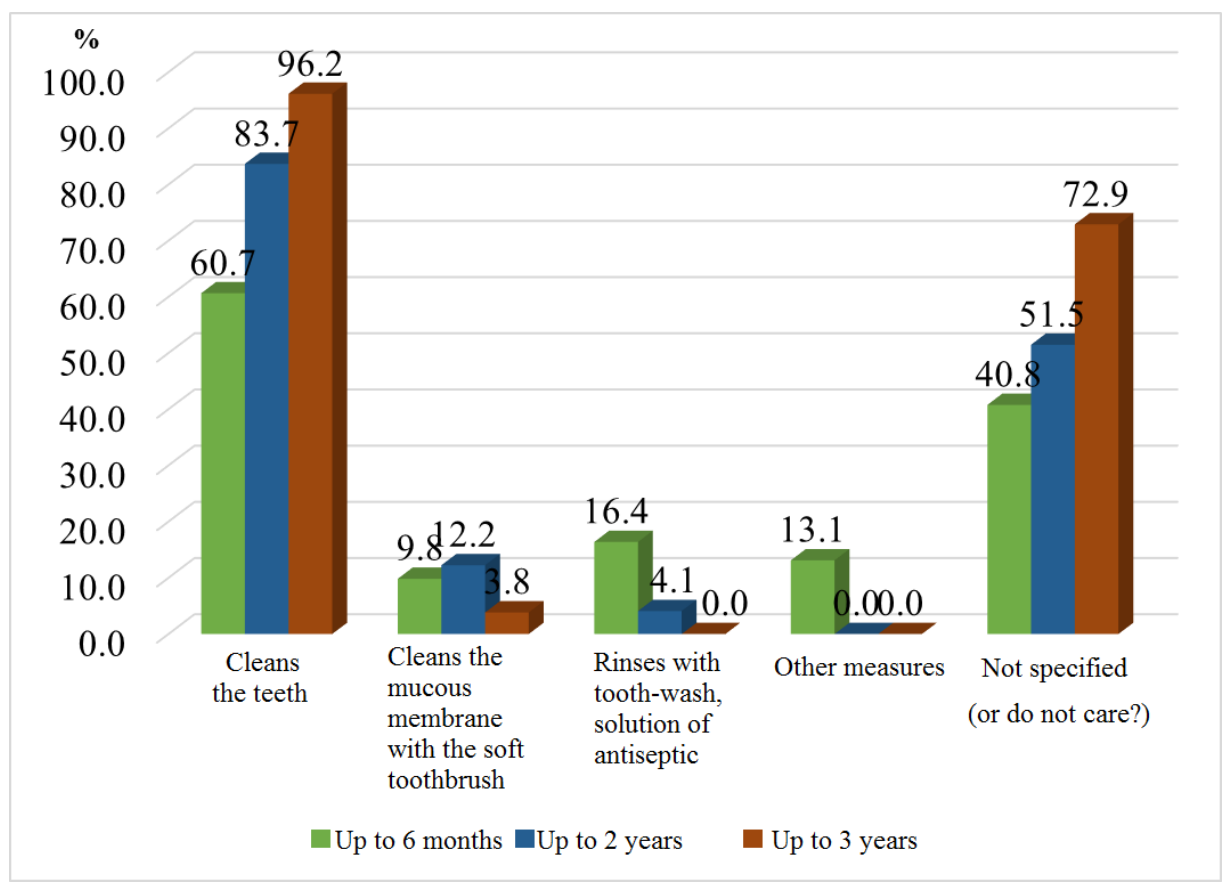

Figure 9. Oral cavity care as a result of the patients' survey.

gienic condition of the oral cavity in persons with removable denture structures. Galician Medical Journal. 2007; 14(3): 121-126.

[4] Rozhko MM, Verbovska RI, Herashchenko SB. Effect of adhesive creams for fixation of complete removable laminar prostheses on the oral cavity mucous membrane. Galician Medical Journal. 2014; 21 (2): 73-77.

[5] Nykyforchyn UR, Kutsyk RV, Rozhko MM, Nykyforchyn RM. Anti-colonization coefficients - are the indicators of local oral immunity. Galician Medical Journal. 1999; 6(2): 99100.

[6] Divnych TYa, Rozhko MM, Kutsyk RV. Change in the oral cavity microflora depending on the period of use of removable denture structures. Galician Medical Journal. 2007: 14(2): 26-29.

[7] Pavlenko OV, Doroshenko OM. Prevention of complications after orthopedic treatment with removable dentures. Ukrayinsky ctomatolohichny almanakh. 2010; 6:39.
[8] Kochkina NA. Modern views and relevance of the influence of partial removable dentures on the ecology of the oral cavity in periodontal tissue disease. Visnyk stomatolohiyi. 2011; $1: 107$.

[9] Zelenova EG. Microflora of the oral cavity: norm and pathology. 2004; 4-26.

[10] Grigoryan AS. The use of valplast base material in the removable dental prosthetics as an alternative to polymethyl methacrylate. Klinicheskaya stomatologiya. 2006; 3:70-75.

Received: 2019-11-22

Revised: 2019-12-13

Accepted: 2019-12-13 